In press at Journal of Experimental Psychology: General. This file is a pre-print and may contain errors or omissions not present in the final published version.

Children's use of polysemy to structure new word meanings

\author{
Mahesh Srinivasan ${ }^{1}$, Catherine Berner ${ }^{1}$, and Hugh Rabagliati ${ }^{2}$ \\ ${ }^{1}$ University of California, Berkeley \\ ${ }^{2}$ University of Edinburgh
}

\begin{abstract}
Author Note
Please address correspondence to: Mahesh Srinivasan, Department of Psychology, University of California, Berkeley. 2121 Berkeley Way \#1650, Berkeley, CA, 94720; 650-823-9488; srinivasan@berkeley.edu

A subset of these data were presented at the $39^{\text {th }}$ Annual Conference of the Cognitive Science Society and the $41^{\text {st }}$ Boston University Conference on Language Development. Raw data and analysis scripts for all experiments reported in this study can be found at https://osf.io/xsh8w/.

Acknowledgements. We thank Megan Merrick, Nicole Hsu, Sophie Waldman and Hannah Peirce for assistance with data collection, and Monica Ellwood-Lowe and Ariel Starr for comments on earlier drafts. This research was supported by an award from the National Science Foundation (SBE-16302040) to MS and an award from the Economic and Social Research Council (ES/N005635/1) to HR.
\end{abstract}




\begin{abstract}
It is well-known that children rapidly learn words, following a range of heuristics. What is less well appreciated is that - because most words are polysemous and have multiple meanings (e.g., 'glass' can label a material and drinking vessel) - children will often be learning a new meaning for a known word, rather than an entirely new word. Across four experiments we show that children flexibly adapt a well-known heuristic - the shape bias - when learning polysemous words. Consistent with previous studies, we find that children and adults preferentially extend a new object label to other objects of the same shape. But we also find that when a new word for an object ('a gup') has previously been used to label the material composing that object ('some gup'), children and adults override the shape bias, and are more likely to extend the object label by material (Experiments 1 and 3). Further, we find that, just as an older meaning of a polysemous word constrains interpretations of a new word meaning, encountering a new word meaning leads learners to update their interpretations of an older meaning (Experiment 2). Finally, we find that these effects only arise when learners can perceive that a word's meanings are related, not when they are arbitrarily paired (Experiment 4). Together, these findings show that children can exploit cues from polysemy to infer how new word meanings should be extended, suggesting that polysemy may facilitate word learning and invite children to construe categories in new ways.
\end{abstract}

Keywords: word learning; polysemy; shape bias; word extension 


\section{Children's Use of Polysemy to Structure New Word Meanings}

Imagine that a child is asked to pick up 'a dax'. The child faces an inductive problem not only in identifying the referent of the new word, but also in extending the word beyond its initial referent, to other 'daxes' (Goodman, 1983; Quine, 1969). The idea that children must solve inductive problems to learn new words has guided decades of research in language development, and has led to the conclusion that children's hypotheses about the structure of new word meanings must be constrained (Markman, 1990, 1991). For example, previous studies show that children exhibit a shape bias when learning a new count noun for a solid object, and expect the noun to extend to other objects that share a similar shape, regardless of whether they otherwise vary in size, material, or color (Landau, Smith, \& Jones, 1988; Smith, Jones, \& Landau, 1996). Heuristics like the shape bias are thought to provide children with intelligent first guesses about the extension of new word meanings, that will later be refined as children accumulate additional evidence and construct adult-like meanings for words (Yoshida \& Smith, 2003).

The task of building a vocabulary is often characterized as one in which children must figure out the meanings of thousands of entirely new words. But this characterization is in many ways inaccurate. In particular, because most words in natural language are ambiguous and express multiple different meanings (e.g., the second edition of the Oxford English Dictionary lists 615,100 definitions for 231,000 words), the more typical scenario is that children will need to learn additional meanings for earlier-acquired words. Despite this fact, surprisingly few studies have explored how a child's prior meaning(s) for a word might affect their inductive hypotheses about a new meaning of that word, or whether learning a new word meaning might lead children to update their beliefs about an older meaning of that word. One notable exception are studies which have suggested that in cases of homophony, where the same word form 
expresses multiple unrelated meanings - e.g., 'bank' to label a financial institution or river - the first meaning that the child learns may hinder their ability to acquire subsequent meanings (Casenhiser, 2005; Doherty, 2004; Mazzocco, 1997).

Interestingly, while it may present challenges for word learning, homophony is actually a surprisingly rare phenomenon. Instead, the most prevalent form of ambiguity in natural language is polysemy - cases in which the same word expresses multiple, related senses (e.g., 'tin' and 'glass' can each label materials or objects made from those materials). Critically, in contrast to homophones, which express unrelated meanings, polysemous senses are related, raising the possibility that children's knowledge of one sense of a polysemous word might actually facilitate - rather than hinder - their acquisition of other polysemous senses (Rabagliati \& Srinivasan, in press; Srinivasan, Al-Mughairy, Foushee, \& Barner, 2017; Srinivasan \& Rabagliati, 2015). Imagine, for example, a child who has learned that 'tin' refers to a kind of material, and who then hears an unfamiliar, solid object made of tin called 'a tin'. Although children typically extend new count nouns for solid objects on the basis of shape (Landau et al., 1988; Smith et al., 1996), the child could guess that this new sense of 'tin' will be importantly related to the material sense of 'tin', and could thus infer that the new object sense of 'tin' will be partly defined by the object's material composition, and not just its shape. They might thus expect other 'tins' to be made of tin, conforming to how adult speakers actually use this word. Similarly, a child that first encounters 'tin' in its object sense might initially expect it to be extended according to shape, but could later update this interpretation upon learning that a different sense of 'tin' can label the kind of material that composes the object. As these examples suggest, polysemy may invite children to construe categories in new ways, shaping their beliefs about their underlying structure and scope. To test this idea, the present studies explore how preschoolers' and adults' 
knowledge of one meaning for a word might interact with their use of word learning heuristics, like the shape bias, to affect their inductive hypotheses about other senses of the word.

\section{Identifying the Referents of New Homophonous and Polysemous Meanings}

As noted above, several studies have explored how homophones are acquired, and have suggested that the first learned meaning of a homophone can interfere with how children acquire additional meanings (Casenhiser, 2005; Doherty, 2004; Mazzocco, 1997).To simulate the conditions in which children learn new homophones, Mazzocco (1997) presented children with stories in which familiar words (e.g., 'rope') were used in new ways to denote novel referents (creating pseudohomophones; e.g., 'Becky pushed the sharp metal rope into the dirt'), and asked children to select the picture that depicted the referent of the critical word from a set of pictures that included the word's new intended, homophonous meaning (e.g., a spade), its familiar meaning (e.g., a rope), and other unrelated distractors. Strikingly, even 7- and 8-year-olds often failed to select the picture representing the intended homophonous meaning (i.e., the spade), and instead often selected the picture corresponding to the familiar meaning of the word (i.e., the rope). Mazzocco (1997) also showed that children's confusion could not be explained by an inability to use the linguistic context to induce a new meaning, since children were readily able to use context to identify the target referent when the critical word was a novel word form: e.g., 'Becky pushed the sharp metal gler into the dirt'.

The pattern of findings reported by Mazzocco (1997) - which has been replicated and extended by other studies (Casenhiser, 2005; Doherty, 2004) - suggests that it may be harder for children to learn a new homophonous meaning for an existing word in their vocabulary than to learn an entirely new word with that same meaning. One explanation for this is that when children are exposed to a new homophone - e.g., the use of 'rope' to refer to a spade - they 
experience a conflict between the familiar meaning of the known word (i.e., rope) and the new intended meaning of that word in context (i.e., spade), perhaps because these meanings are unrelated and inhibit one another (Frazier \& Rayner, 1990). If children have difficulty suppressing the familiar meaning of the word in this situation, they might fail to establish the referent of the new use of the word (i.e., that 'rope' refers to a spade), hindering acquisition of this new meaning (Mazzocco, 1997; Peters \& Zaidel, 1980). The idea that a child's first meaning for a word form competes with a novel, secondary meaning for that word is also consistent with previous evidence that, even when children have learned both meanings of a pair of homophones, they have difficulty retrieving the less frequent meaning of the pair (Campbell \& MacDonald, 1983; Peters \& Zaidel, 1980), unless strong contextual support is provided for this less frequent meaning (Beveridge \& Marsh, 1991; Rabagliati, Marcus, \& Pylkkänen, 2013).

Although the evidence reviewed above suggests that it may be harder for children to learn a new homophone for an existing word than to learn an entirely new word with that meaning (for evidence on the conditions in which children can learn new homophones, see Casenhiser, 2005; Dautriche, Chemla, \& Christophe, 2016), this conclusion may not extend to the acquisition of new, polysemous senses. Indeed, there is psycholinguistic evidence (e.g., Frazier \& Rayner, 1990) that the different senses of polysemous words may not compete and inhibit with one another in the same way that homophones do. Further, because the senses of polysemous words are related, understanding one sense of a polysemous word might offer useful clues to children for learning additional senses of the word (Rabagliati \& Srinivasan, in press; Srinivasan \& Rabagliati, 2015). Thus, as an example, a child could reason that when a word for an animal is used in a context denoting food, it might refer to the meat that is derived from that animal, facilitating their interpretation of how words like 'chicken' and 'turkey' are used. Consistent 
with this, prior studies suggest that - in contrast to the case of homophones - children do not have difficulty establishing reference for a new, polysemous use of a word (Rabagliati, Marcus, \& Pylkkänen, 2010; Srinivasan et al., 2017; Srinivasan \& Snedeker, 2011, 2014). For example, upon learning a novel word for an animal (e.g., that 'tima' labels a chicken), four- and five-yearolds readily shift their interpretation of the label to refer to the meat derived from the animal (e.g., such that 'tima' labels chicken meat; Srinivasan \& Snedeker, 2014), and preschoolers are similarly flexible at shifting new words between representational objects and their abstract content (e.g., 'heavy book' vs. 'interesting book'; Srinivasan \& Snedeker, 2011), physical containers and their contents (e.g., 'wash the pot', 'stir the pot'; Rabagliati et al., 2010), actions and tools enabling those actions (e.g., 'She shoveled the snow' vs. 'She picked up a shovel'; Srinivasan et al., 2017), and more.

Strikingly, children are not only able to understand new uses of polysemous words when presented with them, but also spontaneously anticipate the different ways that polysemous words can be used to refer (Srinivasan et al., 2017). For example, in one study, preschoolers watched an agent use a novel tool to perform an action on a novel substance, and learned a novel verb that labeled the action: 'Look, he's daxing it.' Next, children were shown an image of the novel tool and an image of the novel substance, and were asked to indicate which was the referent of a second word: 'Show me the lum.' Interestingly, children typically selected the substance rather than the tool, suggesting that they had spontaneously predicted that the tool would be referred to as 'a dax', which in turn blocked the new, second word ('lum') from labeling that tool. Thus, children expected the new word for the action to follow a predictable pattern of English polysemy in which the same word labels an action and the tool that enables it (e.g., 'a hammer' / 'to hammer', 'a shovel' / 'to shovel', etc.). This finding suggests that, once young children have 
learned one sense of a polysemous word, they can anticipate additional uses of that word, in line with existing patterns. This conclusion also converges with findings that children generalize patterns of polysemy in their spontaneous speech innovations: e.g., "Don't broom my mess," “It's trucking”(Clark, 1982; see also Bowerman, 1983).

Importantly, the ability to anticipate additional senses of polysemous words could provide a powerful tool for building a lexicon, because predictable patterns of polysemy are quite common within and across languages (Apresjan, 1974; Copestake \& Briscoe, 1995; Lakoff, 1987; Lehrer, 1990; Ostler \& Atkins, 1992; Nunberg, 1979; Srinivasan \& Rabagliati, 2015; Table 1). This potential learning advantage might help explain why polysemy is ubiquitous in natural language (Pustejovsky, 1995), and a far more prevalent form of ambiguity than homophony. For example, Rodd, Gaskell, \& Marslen-Wilson (2004) report that in the Wordsmyth English dictionary (Parks, Ray, \& Bland, 1998), while only $7.4 \%$ of words can be classified as homonyms, $84 \%$ can be classified as polysemous. It may be easier for children to learn a polysemous lexicon in which words are linked to multiple meanings in predictable ways, than an unambiguous lexicon in which each meaning has its own unique word, or a lexicon in which words have multiple unrelated, homophonous meanings (Rabagliati \& Srinivasan, in press; Srinivasan \& Rabagliati, 2015; Srinivasan et al., 2017).

Table 1. Patterns of Polysemy in English

Patterns and Participating Words

Material for Artifact

(glass, tin, iron, etc.)

Animal for Meat

(chicken, turkey, fish, etc.)
Examples

That window is made from glass/

She drank water from the glass

The chicken seemed thirsty/

The chicken was delicious 
Object for Representational Content

(book, magazine, DVD, etc.)

Container for Contents

(pot, bowl, box, etc.)

Body Part for Object Part

(leg, arm, back, etc.)

Artist for Product

(Picasso, Camus, Mozart, etc.)

Place for Institution

(White House, Wall Street, City Hall, etc.)

Instrument for Action Involving Instrument

(shovel, hammer, rake, etc.)

Substance for Placing Substance at Goal

(butter, salt, water, etc.)
The book is too big to fit in my bag/

The book is thought-provoking

She scrubbed the pot with a sponge /

She stirred the pot

He injured his leg last year/

That table has a broken leg

Picasso was born in Spain /

The museum just acquired a Picasso

The White House is being renovated /

The White House made an announcement

She has a new shovel /

She will have to shovel the snow

He bought some butter from the store/

He likes to butter his bread

\section{Word Extension and the Shape Bias}

Critically, although the studies reviewed above suggest that it may be easier for children to guess the referent of a new word meaning if they know a prior, polysemous sense for that word, they say little about how children's knowledge of a prior word sense might affect their hypotheses about the structure of a new word meaning, and thus, whether polysemy may affect how children construe new categories. For example, a child might expect that a word for an action, like 'daxing', will also refer to a tool that enables that action, but this still leaves open how children will think the word for the tool should be extended: i.e., which other objects can be called 'daxes'. As described before, researchers have often noted that the extension of a new word meaning will always be under-determined by the evidence that children have observed 
(Goodman, 1983; Quine, 1969), which has led to the conclusion that children's hypotheses about the structure of new word meanings must be constrained (Markman, 1990; Markman, 1991).

One heuristic that might guide children's early word extension is the aforementioned shape bias: Many studies have shown that, when presented with a solid object, and a word embedded in a count noun context (e.g., 'This is a dax' or 'Here is another $d a x$ '), adults and children as young as age two will selectively attend to the shape of the object, leading them to extend the word to other objects that match in shape, and ignore variation in material, color, and size (Landau et al., 1988; Smith et al., 1996). This bias - which appears to accelerate children's acquisition of words (Smith, Jones, Landau, Gershkoff-Stowe, \& Samuelson, 2002) - increases with age and remains strong in adulthood (Landau et al., 1988). By some accounts, the shape bias reflects a learned attentional bias that emerges from a correlation between linguistic cues and perceptual properties of objects (Smith, 2000; Smith et al., 1996; Smith et al., 2002; Smith \& Samuelson, 2006). Specifically, because many of the first count nouns that English-learning children acquire refer to categories of solid objects that are well-organized by shape (e.g., ball, cup, etc.; Samuelson \& Smith, 1999), children might form a higher-level generalization and expect object labels in general to be structured by shape, facilitating the acquisition of additional count nouns.

Of course, the shape bias is not sufficient for acquiring adult-like meanings for many nouns, since noun meanings can be structured by criteria beyond shape, including other perceptual properties (such as material, color, texture, etc.) and more abstract and conceptual criteria (such as intended function, essence, etc.; Bloom, 2002). With this in mind, proponents of the attentional learning account argue that the shape bias is best viewed as a heuristic that can provide children with first guesses about the extension of new words, that will be refined as 
children receive more evidence about the properties that are relevant for specific word meanings (Yoshida \& Smith, 2003), or as children develop additional attentional biases that supersede the shape bias within specific contexts (e.g., such that words for animates are extended by shape and texture; Jones, Smith, \& Landau, 1991). Meanwhile, critics of the attentional learning account have argued that children rely less on shape when conceptual criteria for word extension are made more clear (Booth \& Waxman, 2002; Booth, Waxman, \& Huang, 2005; Diesendruck, Markson, \& Bloom, 2003; Kemler Nelson, Russell, Duke, \& Jones, 2000), and have used these findings to suggest that the shape bias is not a product of attentional learning but instead reflects children's understanding that shape provides a good cue to category membership.

\section{The Present Studies}

Our goal in the present studies was not to re-litigate why children exhibit a shape bias, but to instead explore how polysemy might interact with this bias, which is among the most robust phenomena in language development. In particular, we were interested in whether children's knowledge of one sense of a polysemous word might affect their inductive hypotheses about how another sense for that word should be extended, leading them to shift away from the shape bias in some cases.

Our studies were motivated by the observation that many historically-derived meanings of polysemous words have been structured in part by the historically-antecedent meanings of these words. For example, words like 'glass', 'tin', and 'iron' initially only labeled kinds of materials in Old English, and were later extended to denote specific artifacts made from those materials (Harper, 2001): e.g., drinking 'glass' (first recorded in the early $13^{\text {th }}$ century), hour 'glass' (1550s), eye 'glasses' (1660s), 'tin' container (1795), clothes 'iron' (1610), golfing 'iron' (1842). The influence of the material meanings of these words on their artifact meanings can be 
observed in the present day. In particular, although technological and cultural changes have led to semantic drift of the artifact meanings of some words away from their material meanings e.g., today, one can refer to eye 'glasses' that are made of plastic - it is clear that the artifact meanings of many of these words are extended not simply by shape and function, but also by material. For example, a cup that is made of brass cannot be called a 'glass', nor can a box made of wood be called a 'tin'. The primary goal of the present studies was to explore whether polysemy can help children infer these extensional patterns; if a new word for a material is also used to denote an object made from that material, will children expect the object label to be extended in part by material? At stake in this question is whether polysemy may invite children to construe categories in new ways.

In critical trials of Experiments 1 through 3, children and adults were taught a novel name for a material, which was embedded in mass noun syntactic frames (Figures 1, 4). They were also presented a novel object made from the same material, which was labeled using count noun syntactic frames, either using the same word form as the material name ("Polysemy condition"; e.g., the material was referred to as 'some gup' and the object as 'a gup') or using a new, distinct word form ("Unambiguous condition"; e.g., the material was called 'some zev' and the object was called 'a gup'). Then, participants in both conditions were asked to extend the object label, which we assessed using a forced-choice task in Experiments 1 and 2, and a more open-ended sorting task in Experiment 3. Given previous evidence that children and adults expect new count nouns for solid objects to be extended according to shape, one possibility was that participants in both conditions would exhibit a shape bias, and prefer to extend the new object label to other items that match in shape, ignoring variation in material. However, we hypothesized that observing that a word for a material was also used to label an object made from that same 
material would lead participants to construe this material as essential to the object label's meaning; thus, participants in the polysemy condition should take material into account when extending the object label.

Some evidence related to our hypothesis comes from a set of studies conducted by Yoshida and Smith (2003). In the critical trials of one of their studies, one of two familiar object words was used to label a novel object whose form lay somewhere between the two familiar object categories: For instance, a green ovoid object was labeled either as an 'egg' or as a 'pickle'. Children were then asked to extend the word for the novel object further, to either an object matching only in shape (but not material or color), an object matching only in material (but not shape or color), or an object matching only in color (but not shape or material). Interestingly, children showed a shape bias when the novel object was labeled with a familiar name that adults judged to be strongly associated with a characteristic shape (e.g., 'egg'), but were more likely to extend according to material or color when the familiar name was more strongly associated with these other dimensions (e.g., 'pickle'). From these and other findings, Yoshida and Smith (2003) argued that when children hear a known word, this causes them to allocate attention to the particular dimensions that are associated with that word (shape for words like 'egg', material for words like 'pickle'), even when the word has been applied to a novel object.

Although the findings of Yoshida and Smith (2003) are consistent with our proposal, it is unclear how they relate to phenomena like polysemy and homophony, and what they might predict about how knowledge of one meaning of a word might affect the extension of a new, distinct meaning for that word. This is because the novel objects used in Yoshida and Smith's study were designed so that they could be interpreted as atypical referents of a known word 
meaning. For instance, the labels 'egg' and 'pickle' were applied to an object that had an egglike shape and pickle-like color, and could thus plausibly be interpreted as an atypical 'egg' or 'pickle'. In contrast, however, the referents of different homophones and of different senses of polysemous words cannot typically be considered to be members of a common category: A baseball 'bat' is not an atypical animal 'bat', nor is a piece of 'glass' material an atypical drinking 'glass'. Thus, it remains to be seen whether knowledge of one meaning of a word can influence children's beliefs about how a new, distinct polysemous sense (Experiments 1 through 3) or homophonous meaning (Experiment 4) should be extended, which was the focus of our studies.

\section{Experiment 1}

Using a two-alternative forced-choice task, Experiment 1 provided a test of whether children's and adults' extension of a new word for an object is affected by their knowledge that this word also refers to the material that constitutes the object. Across three conditions, we tested separate groups of English-speaking three-year-olds, four-year-olds, and adults.

In the training phase of critical trials of the polysemy and unambiguous conditions, participants first learned a mass noun for a novel material, and were then shown a novel standard object made from the same material (Figure 1). This standard object was labeled using a count noun; This label either reused the same word form as the material name (polysemy condition, e.g., the material was referred to as 'gup' and the object as 'a gup'), or used a new, distinct word form (unambiguous condition, e.g., the material was called 'zev' and the object was called 'a gup'). In the test phase, participants in both conditions were then asked to extend the name of the object to one of two test items, using count noun syntax (e.g, 'Can you point to a gup?'): One test item matched the first object in material but not in shape (material-match object), and the other 
matched the first object in shape but not in material (shape-match object). We expected that participants in the polysemy condition might be more likely to select the material-match if their knowledge of the prior, material sense of the label influences their hypotheses about the extension of the object name. In contrast, despite observing the same sequence of stimuli, we expected that participants in the unambiguous condition might show a shape bias, because the object name was novel for them and did not have a prior material sense.

One issue regarding the interpretation of the polysemy condition was whether participants in this condition - who were taught that the same word form labels a material and object in training - would truly learn distinct material and object senses of a single polysemous word. Alternatively, participants in that condition might assume that the word refers to the material both when it is initially used to refer to the material, and also when it is subsequently used to refer to the object (e.g., participants might construe the object as an instance of the material; Gelman \& Bloom, 2000; Prasada, Ferenz, \& Haskell, 2002). This alternative presents a potential confound to our hypothesis because it similarly predicts that participants in the polysemy condition should select the material-match object at test (since it is the only item that is composed of the relevant material) even though they have not learned distinct material and object senses.

We took two steps to address this issue. First, we designed our procedure such that it would be unlikely for participants to interpret the word as having a material or substance meaning when it was re-used to label the object. For instance, the object stimuli that we presented in training (see Figure 1) were unlikely to be construed as substances because they were solid and complex (i.e., their outline was defined by multiple points and sides; Imai \& Gentner, 1997; Li, Dunham, \& Carey, 2009) and had regular shapes (i.e., straight edges and 
curves with constant or smoothly changing curvature; Prasada, Ferenz \& Haskell, 2002).

Previous research has consistently found that such stimuli are treated as objects by both children and adults (Li et al., 2009; Prasada et al., 2002), such that words labeling these stimuli are more likely to be quantified by number (as opposed to area or volume) and extended by shape rather than material (Barner \& Snedeker, 2006; Imai \& Gentner, 1997; Soja, 1992; Soja, Carey, \& Spelke, 1991; Subrahmanyam, Landau, \& Gelman, 1999). Moreover, we introduced the new object word meanings by using count syntax and subsequently referred to the objects using these count nouns; Prior studies indicate that children and adults assume that count nouns will label individuals like objects, such that they are quantified by number and extended by shape (Barner \& Snedeker, 2005, 2006).

Our second step was to include an additional object vs. material control condition to test whether participants had learned distinct object and material senses. Participants in this third condition received the same training as in the polysemy condition, but the choice items presented at test were a material-match object and a portion of the material itself (Figure 1). We reasoned that if participants have learned distinct material and object senses of the new word in training, and recognize that they have been asked to extend the object sense of the word at test, they might expect this label to be extended to the material match object, and not to the portion of the material, since the former is the more prototypical object. In contrast, if participants treat the material and object presented in training as exemplars of a single word that refers to a material, they might select the portion of material above chance (since it is the more prototypical exemplar of material), or perhaps select between the material-match object and the portion of material at chance (since they both contain the relevant material).

\section{Method}


Participants. We enrolled 100 children, including 49 3-year-olds (Mean age = 3;6 years; range $=[3 ; 0-3 ; 11] ; 24$ girls $)$ and 514 -year-olds $(M=4 ; 5[4 ; 0-4 ; 11] ; 26$ girls $)$, as well as 48 adults $(M=22[18-50] ; 26$ women), all of whom were recruited from the Berkeley, California area. Participants in each age group were randomly assigned to the polysemy condition (17 3year-olds, $M=3 ; 7[3 ; 0-3 ; 11] ; 164$-year-olds, $M=4 ; 7$ [4;0-4;11]; 16 adults, $M=24$ [18 50]), the unambiguous condition (16 3-year-olds, $M=3 ; 7$ [3;0-3;11]; 18 4-year-olds, $M=4 ; 5$ [4;0 - 4;11]; 16 adults, $M=22$ [18 - 31]), or the material vs. object condition (16 3-year-olds, $M$ $=3 ; 6[3 ; 0-3 ; 11] ; 174$-year-olds, $M=4 ; 5[4 ; 0-4 ; 10] ; 16$ adults, $M=21[19-31])$. An additional 16 adults $(M=21[19-31] ; 8$ women $)$ were also recruited and tested in a replication of the material vs. object condition. English was the primary language spoken by all participants. Children were tested in lab, or recruited from and tested at local preschools and museums; Adults were recruited from and tested on the UC Berkeley campus. Children were given a small gift for participating, and adults received either course credit or a small gift. Sixteen additional children participated but were excluded due to parental interference $(n=3)$, experimenter error $(n=1)$, or for failing two out of three catch trials that were administered at the end of the task $(n=12$; described below). Three adults were also excluded due to experimenter error. All participants were tested individually by a female experimenter. All experiments reported here were approved by the local Institutional Review Board at UC Berkeley.

Materials and procedure. Participants were seated across from the experimenter at a table. Participants received three warm-up trials, followed by four critical trials, and finally, three catch trials. These trials were administered to participants in one of two sequences, and we also counterbalanced the left-right placement of choice objects on each trial, resulting in four item orders overall for each condition. 
Warm-up Trials. The warm-up trials were designed to help participants understand the task. The stimuli consisted of three sets of toy animals, and each set included two identical animals from a target category and one animal of a different kind (e.g. two bears and a horse). We included only animals that would be familiar to young children. In each warm-up trial, the experimenter placed one of the animals from the target category on the table, and named it (e.g. "Here is a bear!"), and then placed the remaining two animals on the table and asked the participant to point to the other matching animal (e.g. "I want another bear. Can you point to a bear?").

Critical Trials. Each of the four critical trials consisted of a training phase, and a test phase (Figure 1). In the training phase of each trial, the experimenter labeled small pieces of a novel material in a jar with a novel word, using mass syntax: 'This stuff is called [gup]. This stuff is called [gup]. I have half a jar of [gup] here.' To give additional cues that the referent was a material, the experimenter then stirred the material with a spoon and scooped some of it out of the jar and put it onto the lid of the jar: 'Look, I am stirring the [gup] and now I am scooping some of it out of the jar.' The experimenter then moved the jar and lid full of material off of the table, out of sight. Next, the experimenter brought out the standard object, which was an object that was composed of the material, and labeled it using count syntax: 'Now look at this thing! This thing is called a [gup]. This thing is called a [gup].' To further illustrate that the referent was an object, the experimenter also attributed a vague function to the standard object: e.g., 'I have two gups and I use them in my garage.' Critically, the name given to the material was the same as that given to the standard object in the polysemy and object vs. material conditions (e.g., both were called 'gup'), but was different in phonological onset and rime in the unambiguous condition (e.g., the material was called 'zev' and the standard object was called 'gup'). 
After moving the standard object to the edge of the table, the experimenter placed the two test items in front of the participant, and asked them to extend the label for the standard object to one of these two test items, using count noun syntax: 'Okay, so now we know what [gups] are! And now it's your turn! I want another [gup]. Can you point to a [gup]?' In the polysemy and unambiguous conditions, the test items were a material-match object (an object that was made out of the same material as the standard object, but of a different shape) and a shape-match object (an object that was the same shape as the standard object but of a different material). For the object vs. material condition, the experimenter brought back out the lid full of material and placed it along with the material-match object in front of the participant.
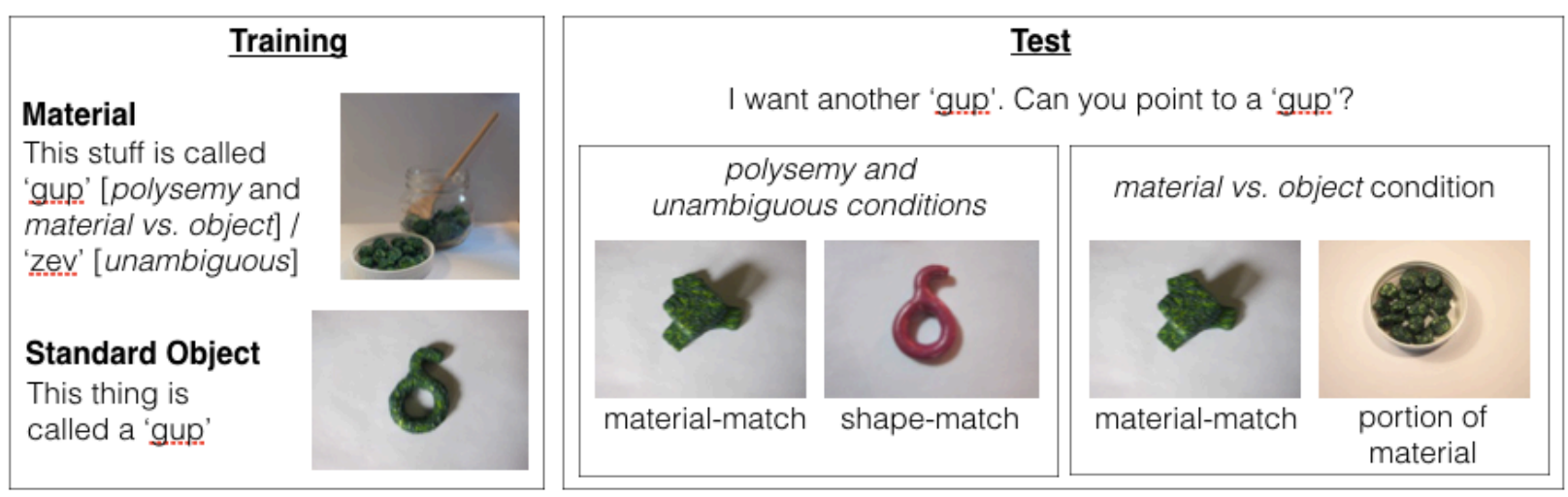

Figure 1. An example of a critical trial from the polysemy, unambiguous, and material vs. object conditions of Experiment 1.

Catch Trials. Finally, participants completed three catch trials at the end of the task to ensure that they had sustained their attention throughout the study. In these trials, the experimenter labeled a first novel object with a novel word, and then asked participants to point to which of two subsequent objects could be labeled by the word. One of the choice objects was identical to the first object, and the other differed in shape and material. Participants who failed to correctly respond on at least two out of the three catch trials were excluded. 
Analyses. We used Bayesian mixed effects logistic regressions (fit using the brms package in R) to separately model the choices of children and adults. Our dependent variable was participants' choice of the material-match object (as opposed to the shape-match object in the polysemy and unambiguous conditions; and as opposed to the portion of material in the material vs. object condition). Condition was dummy coded so that the unambiguous condition was set as the reference level against which responses in the other two conditions was compared. To compare performance in each condition to chance, we also fit individual logistic regressions to each condition that only included an intercept term, and assessed if that term was different from zero. All models included random intercepts for each subject, but did not include random intercepts for the four items, as models frequently failed to converge when they were included (due to partial separation, see below).

We used Bayesian analyses because our data had characteristics that impede fitting logistic regressions using standard maximum likelihood methods. In particular, participants often did not show variability in their responses (e.g., selecting the shape match on every trial); a phenomenon known as separation that can cause mis-identification and over-estimation of model parameters. To account for this, our Bayesian models included conservative normal (0, 2.5) priors on fixed effect regression coefficients, and normal $(0,1)$ priors on random effect estimates of subject variance. In lieu of $p$ values, we report $95 \%$ credible intervals, and describe predictors as "significant" when these intervals do not include zero.

Preliminary analyses of the children's data also included a predictor for age in months, and its interaction with condition. Since this factor was not significant, it was dropped from subsequent models and is not reported here. Raw data and analysis scripts for all experiments reported here can be found at https://osf.io/xsh8w/. 


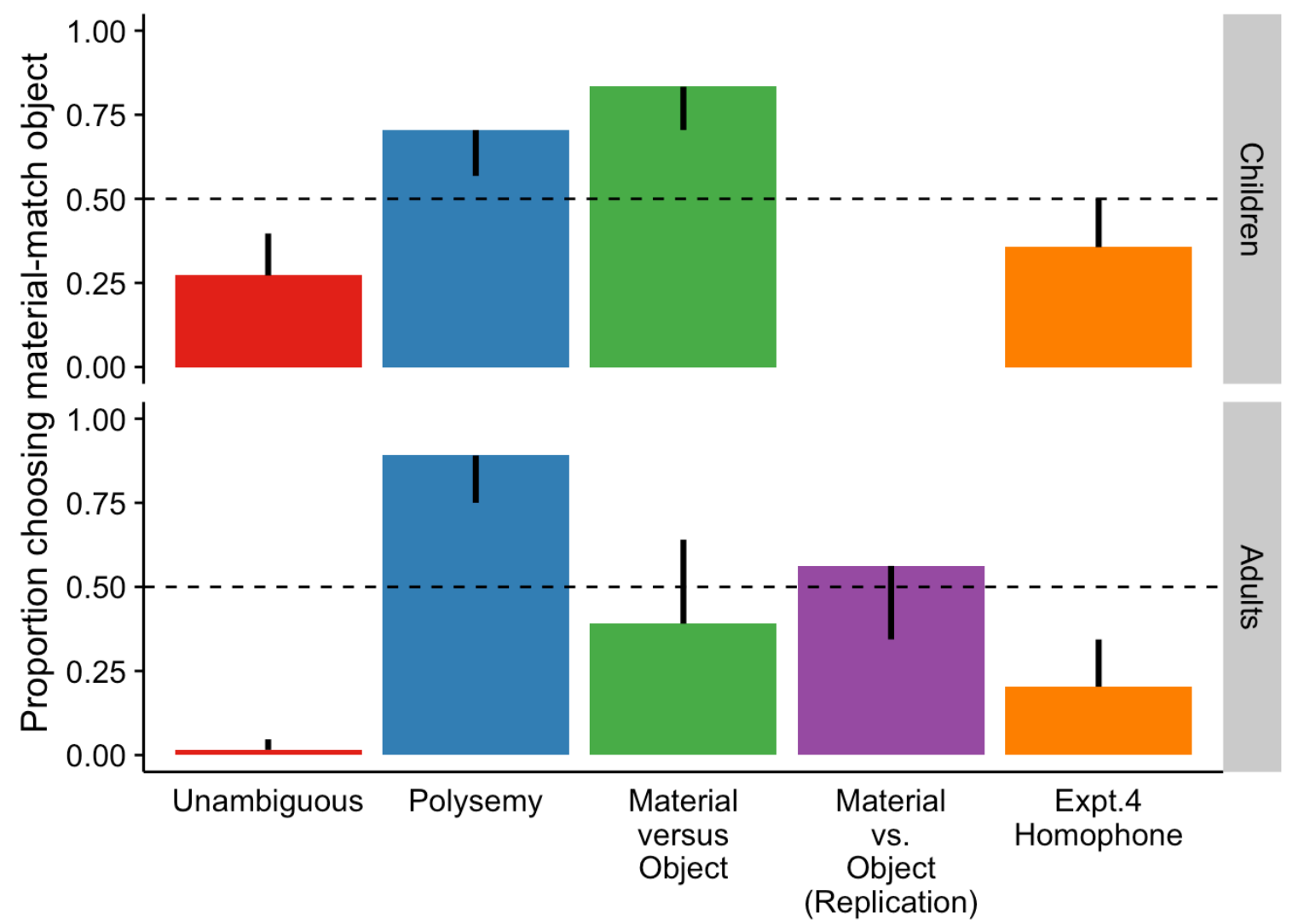

Figure 2. Proportion of trials in which children and adults chose the material-match object in Experiment 1, a subsequent replication of the Material vs. Object condition of Experiment 1, and in Experiment 4 (which was modeled after the Experiment 1 Polysemy condition). Dashed lines represent chance responding (0.5). Error bars show bootstrapped 95\% confidence interval towards chance.

\section{Results}

Figure 2 depicts the proportion of critical trials in which children and adults selected the material-match object across the three conditions. Our model of children's responses indicated that, in the unambiguous condition - when the material and standard object received different names in training - children were significantly less likely than chance to choose the materialmatch object, i.e., they showed a shape bias $(M=0.27,95 \%$ Confidence Interval $=[0.14,0.40]$, 
Beta $=-2.0(S E=0.7), 95 \%$ Credible Interval $=[-3.0,-0.5])$. However, compared to children in the unambiguous condition, children in the polysemy condition - who learned that the same name labeled the material and standard object - were significantly more likely to choose the material match object $(M=0.70[0.56,0.82]$, Beta $=3.4(0.9), 95 \%$ Cr.I. $=[1.8,5.3])$, and in fact chose this object at above-chance levels, indicating that the shape bias had been reversed $($ Beta $=$ $1.8(0.6), 95 \%$ Cr.I. $=[0.7,3.0])$. Evidence from the material vs. object condition indicated that the reversal of the shape bias in the polysemy condition did not stem from an assumption that the critical novel words only referred to material. In particular, in this condition - in which children received the same training as in the polysemy condition - children chose the material-match object significantly more often than children in the unambiguous condition $(M=0.83[0.70$, 0.94], Beta $=5.0(1.0), 95 \%$ Cr.I. $=[3.2,6.9])$, and significantly more often than chance $($ Beta $=$ $3.5(0.8), 95 \%$ Cr.I. $=[2.1,5.4])$. Thus, children in this condition expected the critical novel words to extend to the material-match objects more often than the portions of material, suggesting that they had learned distinct object senses of these words.

Like children, adults in the unambiguous condition also chose the material-match object at below-chance levels, and thus exhibited a shape bias $(M=0.02[0,0.05]$, Beta $=-5.1(1.4)$, $95 \%$ Cr.I. $=[-8.4,-3.0])$. Also similar to the children, adults in the polysemy condition were significantly more likely to select the material-match object than adults in the unambiguous condition $(M=0.89[0.75,1]$, Beta= $7.1(1.2), 95 \%$ Cr.I. $=[4.8,9.5])$, and did so at abovechance levels $($ Beta $=3.6(1.0), 95 \%$ Cr.I. $=[1.9,6.0])$, indicating a reversal of the shape bias. Interestingly, while adults in the material vs. object condition selected the material-match object significantly more than they did in the unambiguous condition $(M=0.39[0.19,0.62]$, Beta $=2.8$ (1.1), 95\% Cr.I. $=[0.4,5.1])$, they did not select the material-match object at above-chance levels 
$($ Beta $=-1.1(0.88), 95 \%$ Cr.I. $=[-2.9,0.6])$, and thus did not select this object significantly more often than the portion of material. In a replication study using the same materials and procedure as in the material vs. object condition, adults also did not select the material-match object significantly more often than chance $(M=0.56[0.36,0.75]$; Beta $=0.33(2.11), 95 \%$ Cr.I. $=[-3.8$, 4.3]; Figure 2). These findings thus leave open whether adults learned distinct object senses of the critical novel words in training.

\section{Discussion}

The findings from Experiment 1 show that while children in the unambiguous condition exhibited the robust shape bias documented in prior work (Landau et al., 1988; Smith et al., 1996), children in the polysemy condition did not, and instead extended the new labels for the objects according to material, rather than shape. The extension by material observed in the polysemy condition was particularly striking because the object labels were presented in count syntax and referred to complex and regularly-shaped objects, factors that have been consistently shown to promote extension by shape in previous studies (Barner \& Snedeker, 2006; Imai \& Gentner, 1997; Li, et al., 2009; Prasada et al., 2002; Soja, 1992; Soja et al., 1991;

Subrahmanyam et al., 1999). Importantly, Experiment 1 also provided evidence that children's tendency to select the material-match object in the polysemy condition was unlikely to reflect that they had only learned a single material meaning of the novel word in training. This would have predicted that children in the material vs. object condition - who received the same training as children in the polysemy condition - should have selected the portion of material at least as often as the material-match object, whereas we found that children selected the material-match object significantly more often than the portion of material. Together, these findings provide evidence that, in the polysemy condition, children's prior knowledge of the material sense of the 
novel word guided their inductive hypotheses about the structure of a distinct object sense for that word, leading them to extend the object sense by material.

Adults showed a similar pattern of results to children in two of the critical conditions, displaying a shape bias in the unambiguous condition that was reversed in the polysemy condition. Unexpectedly, however, adults did not reliably select the material-match object in the material vs. object condition, or in a follow-up replication study. Thus, our results are not decisive with respect to whether adults in the polysemy condition extended the novel word for the object by material because they had learned a prior, distinct material meaning for the word that affected their beliefs about structure of the object meaning, or because they had instead only learned a single, material meaning for the word in training. We return to this issue in the General Discussion.

\section{Experiment 2}

In Experiment 1, both children and adults were more likely to override the shape bias and extend a new word for an object by material when they had previously learned that it could also label the material that the object was made from (polysemy condition), compared to when they had learned separate labels for the material and object (unambiguous condition). Although these findings suggest that prior knowledge of one use of a polysemous word can constrain learners' interpretations of a new use of that word, they leave open whether the reverse is also true: i.e., whether observing a new use of a polysemous word leads learners to update their interpretations of a previously-encountered use of that word. This question is particularly important when considering how children might learn polysemous material-object words like 'glass', 'tin', and 'iron'. Specifically, because children might be exposed to and learn the object-referring senses of these words (e.g., that 'glass' labels a drinking vessel) before their material-referring senses (e.g., 
that 'glass' labels a kind of material), it is important to test whether exposure to a new material sense of a novel word can lead learners to revise their interpretation of a previously-encountered object sense of the word, e.g., leading them to structure the object sense in part by material, as opposed to by shape alone.

Experiment 2 addressed this question using the same methods as Experiment 1, with one critical change: Participants in Experiment 2 first learned a novel count noun for the standard object, and were subsequently shown the material that the object was composed of, which was labeled using a mass noun (i.e., reversing the order of presentation from Experiment 1). As before, we manipulated whether the standard object and material were given the same label (polysemy condition, e.g., the material was referred to as 'gup' and the standard object as 'a gup') or distinct labels (unambiguous condition, e.g., the material was called 'zev' and the standard object was called 'a gup'). Then, in the test phase, the standard object was again referenced (e.g., 'Do you remember this? This is a gup') and participants were asked to extend this object's name (e.g., 'Can you point to a gup?'), either to the material-match object or shapematch object. We expected that if observing a new material sense of a word leads learners to update their beliefs about the structure of a previously-learned, object sense of that word, then participants in the polysemy condition should be more likely to select the material-match object than participants in the unambiguous condition, who should exhibit a shape bias. In contrast, if interpretations of one sense of a word are insulated from later-acquired senses of that word, participants in both the polysemy and unambiguous conditions should show a shape bias.

\section{Method}

Participants. We enrolled 32 4-year-olds $(M=4 ; 7$ [4;0 - 4;11]; 16 girls), as well as 32 adults $(M=21$ [18 - 26]; 16 women). We did not enroll 3-year-olds in this study because we did 
not find significant age effects in our sample of children from Experiment 1. Participants in each age group were randomly assigned to the polysemy condition (16 4-year-olds, $M=4 ; 7$ [4;2$4 ; 11] ; 16$ adults, $M=20$ [18 - 23]) or the unambiguous condition (16 4-year-olds, $M=4 ; 6$ [4;0 4;11]; 16 adults, $M=21$ [19 - 26]). Four additional children participated, but were excluded for failing the catch trials $(n=4)$. All other aspects of the recruitment and compensation were as in Experiment 1.

Materials and procedure. All aspects of the materials, procedure, and analyses were identical to the polysemy and unambiguous conditions of Experiment 1, with the exception that the novel words in the critical trials were first introduced in reference to the objects, before next being introduced in reference to the materials. Then, the experimenter removed the material from the table, brought the standard object back out, and reminded the participant of its name: 'Do you remember this? This is a [gup]. This is a [gup].' Finally, the experimenter initiated the test phase of the trial just as in Experiment 1, by moving the standard object to the edge of the table, placing the two test items (i.e., a material-match object and a shape-match object) in front of the participant, and asking them to extend the label for the standard object to one of these two items. As in Experiment 1, the name given to the material was the same as that given to the standard object in the polysemy condition (e.g., both were called 'gup'), but was different in the unambiguous condition (e.g., the material was called 'zev' and the standard object was called 'gup').

\section{Results}




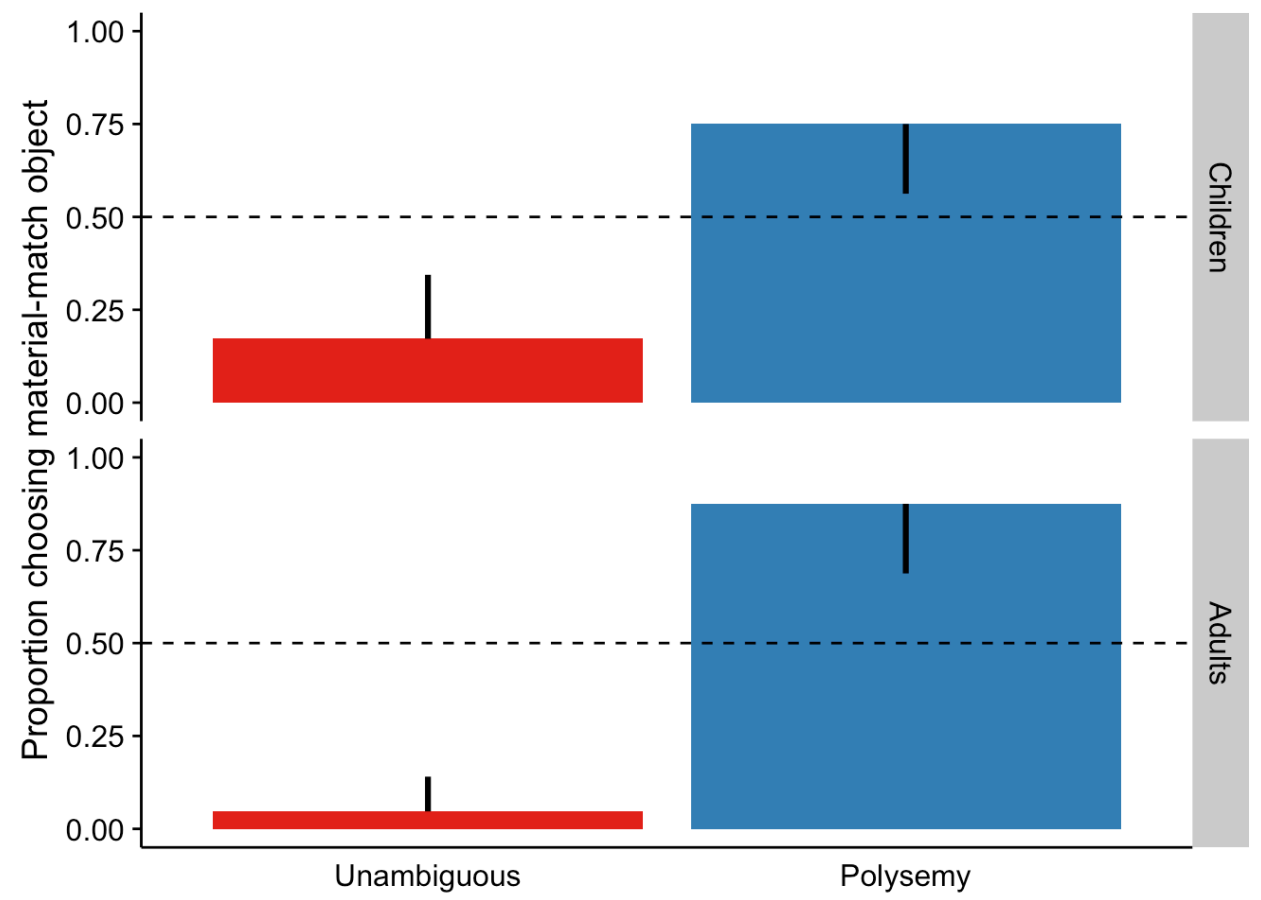

Figure 3. Proportion of trials in Experiment 2 in which children and adults chose the materialmatch object across conditions. Dashed lines represent chance responding (0.5). Error bars show bootstrapped $95 \%$ confidence interval towards chance.

Figure 3 depicts the proportion of critical trials in which children and adults selected the material-match object across the two conditions of Experiment 2. As can be seen, we observed a similar pattern of results to the polysemy and unambiguous conditions of Experiment 1.

Beginning with children, our model indicated that, in the unambiguous condition, children were significantly less likely than chance to choose the material-match object $(M=0.17,95 \%$ Confidence Interval $=[0.03,0.36]$, Beta $=-3.0(S E=0.9), 95 \%$ Credible Interval $=[-5.2,-1.4])$, and thus showed a shape bias. However, compared to children in the unambiguous condition, children in the polysemy condition were significantly more likely to choose the material-match object $(M=0.75[0.56,0.94]$, Beta $=4.7(1.3), 95 \%$ Cr.I. $=[2.3,7.3])$, and chose this object at above-chance levels, indicating that they had overridden the shape bias (Beta $=2.7(1.1), 95 \%$ 
Cr.I. $=[0.7,5.1])$. Together, these findings suggest that exposure to a new material use of the novel word in the polysemy condition led children to update their interpretation of the previously learned object use of the word, such that they now believed that the object use of the word would be structured in part by material, rather than shape alone.

We found the same pattern of results in adults. Like children, adults in the unambiguous condition exhibited a shape bias and chose the material-match object at below-chance levels $(M$ $=0.05[0,0.14]$, Beta $=-4.9(1.3), 95 \%$ Cr.I. $=[-8.6,-2.7])$, but were significantly more likely to select the material-match object in the polysemy condition $(M=0.88[0.69,1]$, Beta= $7.0(1.2)$, $95 \%$ Cr.I. $=[4.8,9.6])$, and did so reliably above chance $($ Beta $=4.3(1.3), 95 \%$ Cr.I. $=[2.1,7.2])$, thus overriding the shape bias. Thus, like the children, adults were able to update their interpretations of the previously-encountered object uses of the novel words upon being exposed to the new, material uses of those words.

\section{Discussion}

Experiment 1 showed that prior exposure to a material use of a novel word constrains both children's and adults' interpretations of a subsequent object use of that word, such that they expect the object name to be extended by material, rather than shape. Experiment 2 extended these findings to show that observing a new material use of a novel word also leads both children and adults to update their interpretations of a previously-encountered object use of a novel word, such that they similarly expect the object name to be extended by material. These findings are significant because they speak to the generality of effects of polysemy on word learning: Just as prior knowledge of one use of a polysemous word can shape interpretations of a new use of that word, exposure to a new use of a polysemous word can lead learners to revise their beliefs of a previously-encountered use of that word. This suggests that, regardless of the order in which the 
senses of a polysemous word are learned, knowledge of one sense of the word can affect interpretations of other senses of that word.

\section{Experiment 3}

In Experiments 1 and 2, both children and adults in the polysemy condition were more likely to extend a word for an object by material when that word had previously been used to label the material, but were more likely to extend it by shape in the unambiguous condition, when the object and material had different labels. However, these findings leave open whether children and adults in the polysemy conditions of Experiments 1 and 2 believed that shape was entirely irrelevant to the extension of the object label, or whether they simply believed that material was more important than shape. One possibility, for example, is that participants in the polysemy conditions of these previous studies privileged material when they were forced to choose between the material-match and shape-match objects, but would have chosen to extend the object label by both material and shape in a less constrained task.

To address this possibility, Experiment 3 employed a more open-ended sorting task. Just as in Experiment 1, four-year-olds and adults were taught a label for a novel material and a label for a novel standard object, and we varied whether or not these two labels were identical. Then, participants were shown an array of new objects that varied in shape, material, and size from the standard object, and were asked to place items that could be labeled by the word for the standard object into a box and the other items into a bowl (Figure 4). Our interest was in whether participants in the polysemy condition would extend words to only items of the same shape and material as the standard object in this more open-ended task.

Participants. We enrolled 32 4-year-old children $(M=4 ; 6[4 ; 0-4 ; 11] ; 16$ girls $)$ as well as 33 adults $(M=20$ [18 - 24]; 17 women). Participants in each age group were randomly 
assigned to the polysemy condition (16 children, $M=4 ; 5[4 ; 0-4 ; 10] ; 17$ adults, $M=20[18-$ 23]) or the unambiguous condition ( 16 children, $M=4 ; 6$ [4;0 - 4;11]; 16 adults, $M=21$ [1924]). Three additional children participated, but were excluded for failing the initial warm-up trials $(n=2$; described below) or due to parental interference $(n=1)$. All other aspects of the recruitment and compensation were as in Experiment 1.

Materials and procedure. The experimenter was seated across from the participant at a table. A plastic transparent box was placed in front of and to the left of the participant, and a plastic transparent bowl was placed in front of and to the right of the participant. Participants received three warm-up trials, followed by four critical trials and a post-test. These trials were administered to participants in one of two sequences, and we also counterbalanced the left-right placement of choice objects on each trial, resulting in four item orders overall for each condition.

Warm-up Trials. The warm-up trials were designed to help participants understand the sorting task. The stimuli consisted of three sets of toy animals. Each set included three animals from a target category and two animals from contrasting categories (e.g., one set included three horses, a cat, and a fish). On each trial, the experimenter brought out a toy animal from the target category and named it: 'Here is a [horse]!' The experimenter then put the animal into the box and told the participant that the box was for the target animals: 'Look, this box is for [horses]. I am putting the [horse] into this box.' The experimenter then took the animal out of the box and placed it on the table in front of the child with the other four animals and asked the participant to place the target animals into the box and all the animals that were not in that category into the bowl: 'Here are some more animals. Some of these are [horses] and some are not [horses]. Can you put all of the [horses] into this box and all of the other animals into this bowl?' Two children 
that did not place all of the target animals into the box on one or more of the three trials were excluded from our analyses.

\section{Material}

This stuff is called

'kiv' [polysemy] /

'lof' [unambiguous]

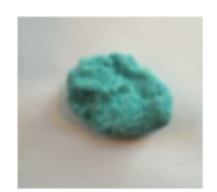

\section{Standard Object}

This thing is

called a 'kiv'

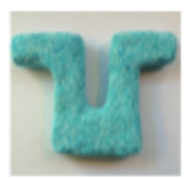

\section{Sorting Test}

Can you put all of the 'kivs' into this box and all of the other things into this bowl?

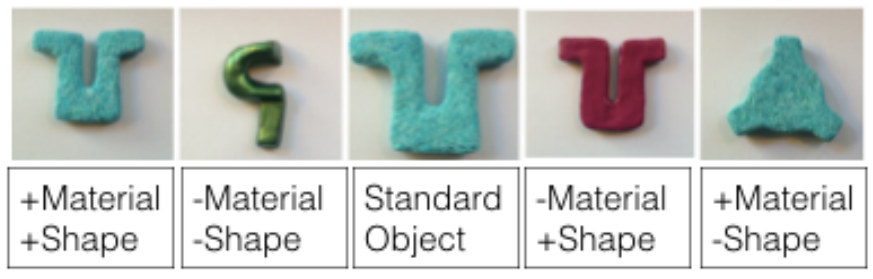

Figure 4. An example of a critical trial from the polysemy and unambiguous conditions of Experiment 3.

Critical Trials. Each critical trial consisted of a training phase, and a test phase (Figure 4). The training was identical to the polysemous and unambiguous conditions Experiment 1, except that we used a new set of novel materials and objects. After participants learned the name of the standard object, the experimenter explained that objects of this kind should be placed into the box, and demonstrated this by placing the standard object into the box: 'Look, this box is for [kivs]. I am putting the [kiv] into this box.' Then, to initiate the test phase, the experimenter took the standard object out of the box, and placed it in front of the child along with four additional test objects. The experimenter then directed the participant to place all items of the target category into the box, and all items that do not belong to the target category into the bowl: 'Here 
are some more things. Some of these are [kivs] and some are not [kivs]. Can you put all of the [kivs] into this box and all of the other things into this bowl?'

The four additional test objects varied in whether they matched the standard object in material and shape (Figure 4). Specifically, the test objects consisted of: (1) A+Material/-Shape object that was made out the same material, but was of a different shape than the standard, (2) a -Material/+Shape object that was the same shape as the standard, but was made out of a different material, (3) a +Material/+Shape object that shared the same material and shape as the standard, but was discernibly smaller, (4) a-Material/-Shape object that was of a different shape and material from the standard, and finally (5) the Standard object itself.

Analyses. We expected that participants in both conditions would sort the +Material/+Shape and Standard objects into the box (treating them as members of the target category), and would sort the-Material/-Shape object into the bowl. Because our hypotheses centered on the classification of the +Material/-Shape and-Material/+Shape objects, we used separate Bayesian mixed effects logistic regressions to model whether participants classified each of these objects as a member of the target category or not. One set of models predicted children's judgments, and another set of models predicted adults' judgments. For each model, our predictor variable was Condition (Polysemy, Unambiguous). As in Experiment 1, we also fit individual logistic regressions to each condition, in order to compare against chance. All models included random intercepts for each subject. Preliminary analyses did not find significant effects of item order, and this factor was dropped from subsequent models.

Finally, to examine whether individual participants were internally consistent in their sorting, we coded the data in terms of whether their responses conformed to one of three categorization strategies. A participant was coded as following a strategy if they made the same 
set of judgments on 3 or more of the 4 test trials. Judgments for a trial were coded as following a Material strategy if the standard and the two objects that matched it in material were classified as members of the target category and placed into the box (i.e., +Material/-Shape object, +Material/+Shape object) and all other objects were placed into the bowl. Meanwhile, judgments for a trial were coded as following a Shape strategy if only the standard and the two objects that matched it in shape were placed into the box (i.e., -Material/+Shape object, + Material/+Shape object), and judgments for a trial were coded as following a Material and Shape strategy if only the standard and the object that matched it in both shape and material $(+$ Material/+Shape object $)$ were placed into the box. Participants that could not be classified as following one of these strategies were coded as following No strategy. We conducted separate chi-square analyses for children and adults, to examine whether the number of participants in each age group that followed these strategies differed across the polysemy and unambiguous conditions. These models dropped from analysis any strategies that were observed too infrequently to have expected counts in the contingency tables of five or more. 


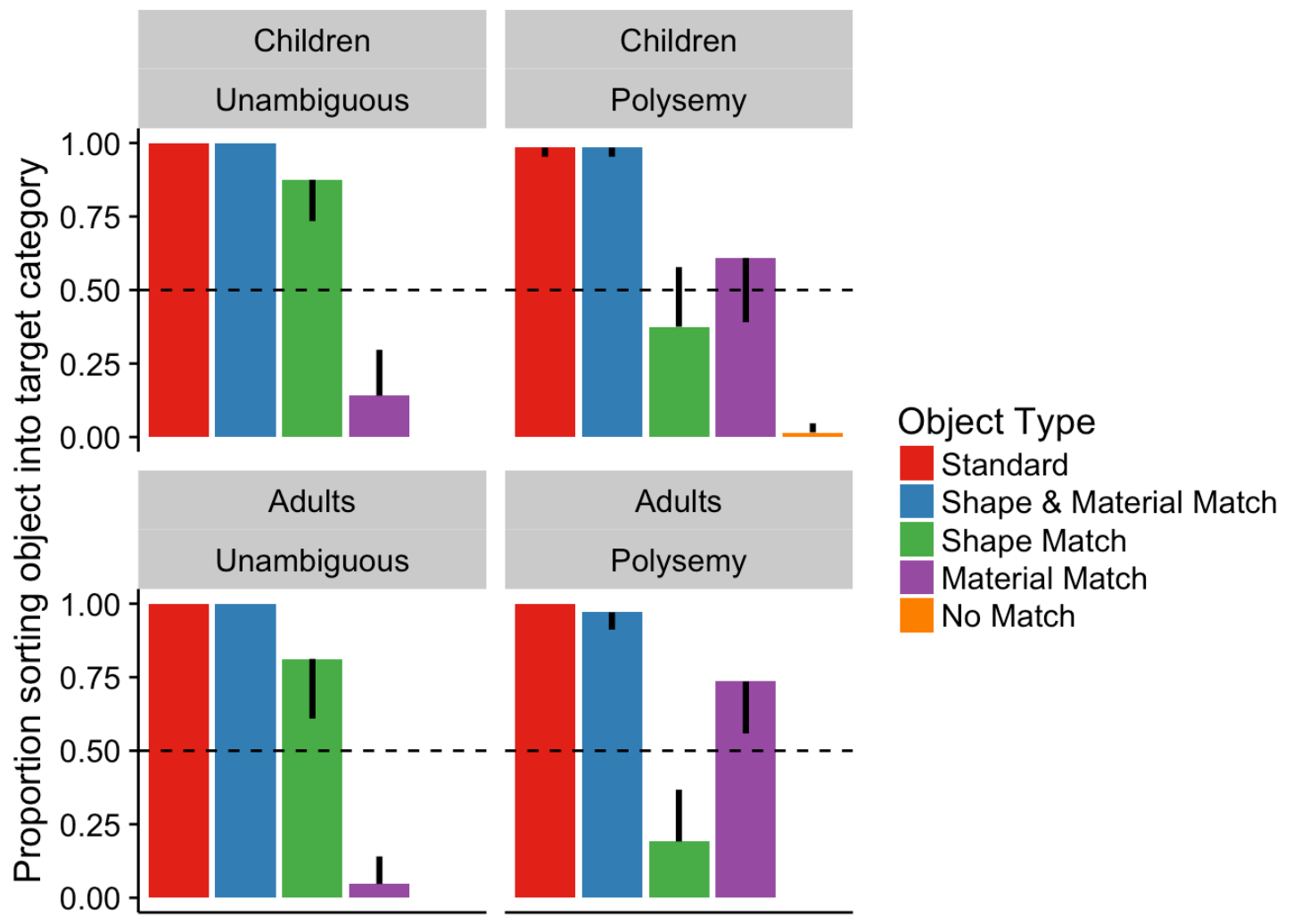

Figure 5. Proportion of trials in Experiment 3 in which children and adults sorted the five test objects into the box, treating them as members of the target category. Dashed lines represent chance responding (0.5). Error bars show 95\% confidence interval towards chance.

\section{Results}

Figure 5 depicts the proportion of critical trials in which children classified each of the test objects as members of the target object kind across the polysemy and unambiguous conditions. Beginning with children, our models detected significant effects of Condition on classifications involving the +Material/-Shape object and the -Material/+Shape object. While children in the polysemy condition did not classify the +Material/-Shape object as a member of the target category significantly more often than chance $(M=0.61[0.39,0.83]$, Beta $=0.94$ $(0.86), 95 \%$ Cr.I. $=[-0.7,2.7])$, they were significantly more likely to do so than children in the unambiguous condition $(M=0.14[0.02,0.30]$; Beta $=2.9(0.9), 95 \%$ Cr.I. $=[1.2,4.9])$, who 
classified this object as a member of the target category significantly less often than chance (Beta $=-3.1(0.9), 95 \%$ Cr.I. $=[-5.1,-1.5])$. In contrast, children in the unambiguous condition classified the -Material/+Shape object as a member of the target category significantly more often than chance $(M=0.88[0.73,0.98]$, Beta $=3.1(0.9), 95 \%$ Cr.I.= $[1.6,5.1])-$ exhibiting a shape bias - and significantly more often than did children in the polysemy condition $(M=0.38$ $[0.17,0.58]$, Beta $=-2.9(0.8), 95 \%$ Cr.I. $=[-4.6,-1.4])$, whose choices did not significantly differ from chance $($ Beta $=-0.9(0.7), 95 \%$ Cr.I. $=[-2.4,0.4])$. Finally, children in both the polysemy and unambiguous conditions almost always sorted the Standard object (Polysemy: $\mathrm{M}=0.98$ $[0.95,1.0]$; Unambiguous: $\mathrm{M}=1.0)$ and the + Material/+Shape object as members of the target category (Polysemy: $M=0.98[0.95,1.0]$; Unambiguous: $M=1.0$ ), and almost never sorted the Material/-Shape object as a category member (Polysemy: $M=0.02$ [0.0, 0.05]; Unambiguous: $M$ $=0.0)$.

Children were also internally consistent in their sorting, and exhibited different sorting strategies across the polysemy and unambiguous conditions (Figure 6). Only two children in the unambiguous condition were classified as following No strategy, and interestingly, no children in either condition followed the Material and Shape strategy - which Experiment 2 was designed to detect. A chi-square analysis - restricted to participants who followed the Material strategy or Shape strategy - indicated that the number of children that followed these strategies differed across the polysemy and unambiguous conditions $\left(\mathrm{X}^{2}(1, \mathrm{~N}=30)=9.85, p<0.005\right)$. In particular, of 16 children in the polysemy condition and 14 children in the unambiguous conditions that followed one of these strategies, 10 of 16 children in the polysemy condition followed the Material strategy (compared to 1 of 14 in the unambiguous condition), and 13 of 14 children of children in the unambiguous condition followed the Shape strategy (compared to 6 of 16 children 
in the polysemy condition). In sum, the findings from children - using the more open-ended task of Experiment 3 - were similar to those from Experiments 1 and 2. Although children in the polysemy condition could have extended the word for the standard object by both material and shape, they often chose to extend this word by material alone, while children in the unambiguous condition typically extended the word by shape.

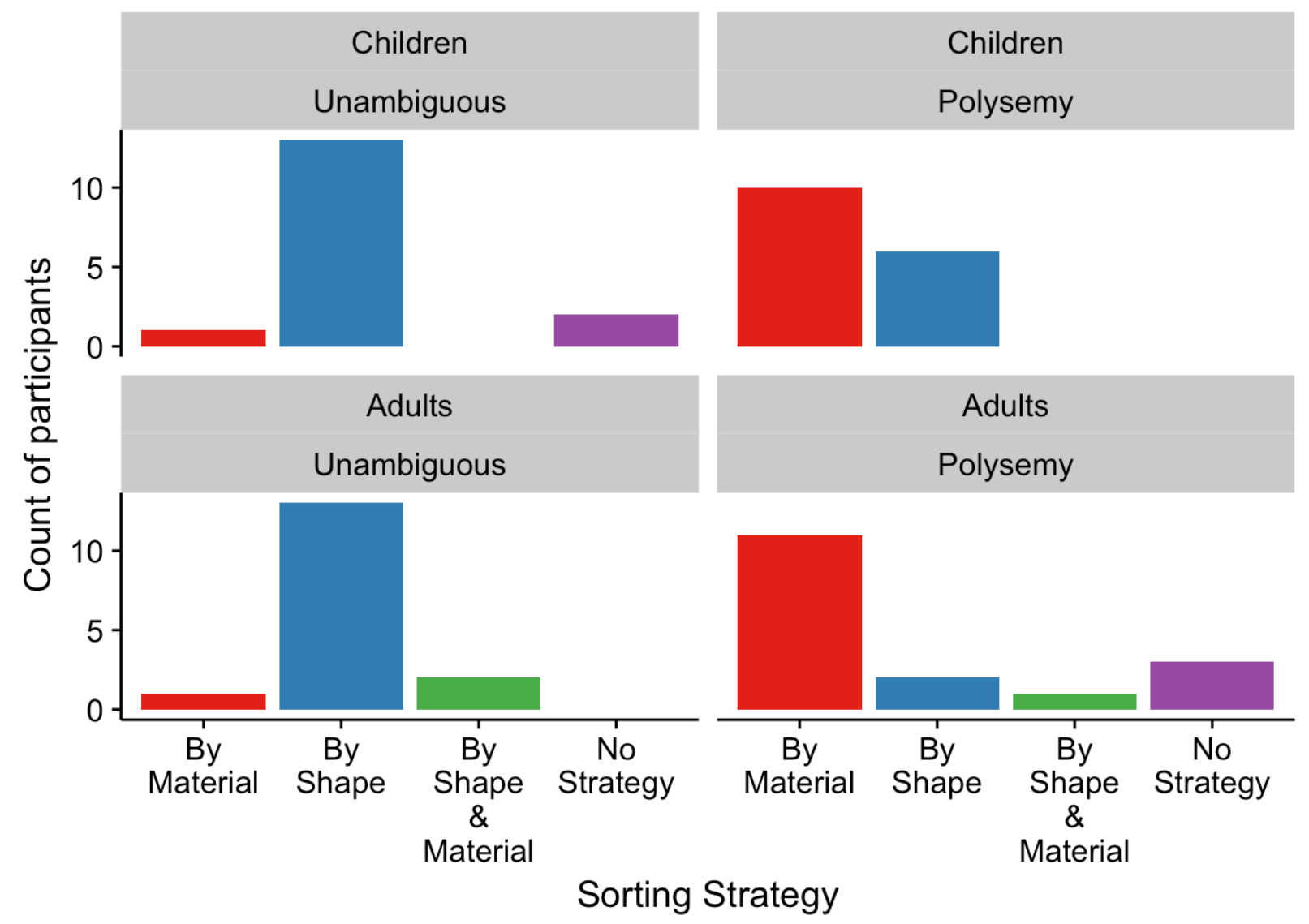

Figure 6. Number of children and adults in the polysemy and unambiguous conditions of Experiment 3 who sorted items according to material, shape, both shape and material, or who exhibited no coherent strategy.

Adults classified the test objects similarly to children (Figures 5, 6). Our models for adults detected significant effects of Condition for classifications involving the +Material/-Shape object $($ Beta $=4.7(1.0), 95 \%$ Cr.I. $=[3.0,6.8])$ and the - Material $/+$ Shape object $($ Beta $=-3.8$ 
(0.9), 95\% Cr.I. $=[-5.7,-2.2])$. These effects reflected that, like children, adults in the Polysemy condition were more likely to classify the +Material/-Shape object as a member of the target category $(M=0.74[0.56,0.90]$, significantly above chance, Beta $=2.0(0.8), 95 \%$ Cr.I. $=[0.6$, 3.9]) compared to adults in the Unambiguous condition $(M=0.05[0,0.14]$, significantly below chance Beta $=-4.8(1.4), 95 \%$ Cr.I. $=[-8.2,-2.6])$, and that adults in the Unambiguous condition were more likely to classify the -Material/+Shape object as a member of the target category $(M=$ $0.81[0.61,0.97]$, significantly above chance Beta $=3.0(1.0), 95 \%$ Cr.I. $=[1.3,5.2])$ compared to adults in the polysemy condition $(M=0.19[0.04,0.37]$, significantly below chance Beta $=-2.6$ (0.7), 95\% Cr.I. $=[-4.5,-1.1])$. No adults in either condition sorted the - Material/-Shape as a member of the target category, and all but one participant sorted the Standard object and the +Material/+Shape object as a member of the target category.

Finally, like the children, adult participants also exhibited different sorting strategies across the two conditions (Figure 6). Only three adults were classified as following No strategy, and three were classified as following the Material and Shape strategy (one in the polysemy condition, two in the unambiguous condition). A chi-square analysis - restricted to participants who followed the Material strategy or Shape strategy - indicated that the number of adults that followed these strategies differed across the polysemy and unambiguous conditions $\left(\mathrm{X}^{2}(1, \mathrm{~N}=\right.$ $27)=16.39, p<0.001)$. In particular, of 13 adults in the polysemy condition and 14 adults in the unambiguous condition that followed one of these strategies, 11 out of 13 in the polysemy condition followed a Material strategy (compared to 1 out of 14 in the unambiguous condition), and 13 out of 14 in the unambiguous condition followed a Shape strategy (compared to 2 of 13 in the polysemy condition).

\section{Discussion}


Experiment 3 provided converging evidence with Experiments 1 and 2, using a less constrained task. As in these previous studies, we found that both children and adults exhibited a shape bias when extending a new word for an object that did not have another meaning (unambiguous condition). However, both children and adults were comparatively more likely to extend the object word by material when it had also been used to label the material that the object was composed of (polysemy condition). Critically, even though the task of Experiment 2 was open-ended enough for participants in the polysemy condition to extend the label for the object by both material and shape, no children and only one out of sixteen adults followed this strategy. These findings suggest that prior knowledge that a word can refer to a material leads both children and adults to base their extension of a new object use of that word on a single feature - material - rather than on a combination of multiple features, like shape and material. In some ways, this reliance on material is surprising, given that the artifact senses of polysemous material words like 'glass' and 'tin' refer to specific kinds of objects that are defined in part by their function and shape, and not by their material alone: e.g., not everything made of glass can be called a 'glass'. We return to this topic in the General Discussion.

\section{Experiment 4}

Taken together, Experiments 1 through 3 provide strong evidence that, when a word that has labeled a novel material has also been used to label a novel object made from that material, learners override a default shape bias and are more likely to expect the object label to be extended by material. But why - and under what circumstances - might knowledge of one word meaning affect inductive hypotheses about the structure of another word meaning? One possibility is that knowledge of one word meaning affects children's extension of another word meaning only when they can perceive that the two meanings might be related. By this account, 
children in the polysemy conditions of Experiments 1 through 3 may have extended the label for the standard object ("a kiv") by material because they could actually see that this object was composed of the same kind of material that the word had also labeled ("some kiv"), leading them to guess that the object meaning might be structured by material. This hypothesis predicts that if children learn a word for a material ("some kiv") that is then used to label an object that is composed of a different material ("a kiv"), they may not think these two uses of the word are related - e.g., they might instead think that they are unrelated homophones - leading them to treat the object label like any new count noun for a solid object, and extend it by shape rather than material.

However, an alternative possibility is that any application of a known word to a novel object may affect word extension, even if the new use of the known word is ostensibly unrelated to its prior meaning. Recall that Yoshida and Smith (2003) found that when children heard a novel object labeled as an 'egg', they subsequently extended that object name by shape, but when children heard the same novel object labeled as a 'pickle', they extended by material. From these findings, Yoshida and Smith (2003) proposed that hearing a known name causes children to automatically allocate their attention to the dimensions associated with that name, thereby affecting subsequent word extension. This proposal opens the interesting possibility that the effects of polysemy documented in Experiments 1 through 3 may not depend on whether children can perceive a relationship between the two senses of the novel words. Instead, when children hear a word that previously has referred to a material now also used to refer to an object, they could automatically attend to the material dimension of that object - affecting their extension of the object label - even when the object is composed of a different material than the 
word had previously labeled (i.e., even when the object and material uses of the word are best described as 'homophones').

Using the forced-choice method of Experiments 1 and 2, Experiment 4 tested the predictions of these different accounts by teaching participants a new label for a material (e.g., 'some kiv') and then teaching them that the label could also apply to an object that was composed of a different material ('a kiv'; Figure 7). Of interest was whether participants would choose to extend the new object label by material, or would instead show a shape bias. In addition to adults, we tested separate samples of 3-year-olds and 4-year-olds, to determine whether the effects of polysemy documented in our Experiments 1 through 3 can be explained in similar ways for children of these different ages.

\section{Method}

Participants. We enrolled 33 children, including 17 3-year-olds (Mean age $=3 ; 7$ years; range $=[3 ; 1-3 ; 11] ; 10$ girls $)$ and 17 4-year-olds $(M=4 ; 4[4 ; 0-4 ; 10] ; 9$ girls $)$, as well as 16 adults $(M=22[18-35] ; 8$ women). Six additional children participated but were excluded due to failing the catch trials. All other aspects of the recruitment and compensation were as in Experiment 1.

Material
This stuff is called
'gup'
Standard Object
This thing is
called a 'qup'

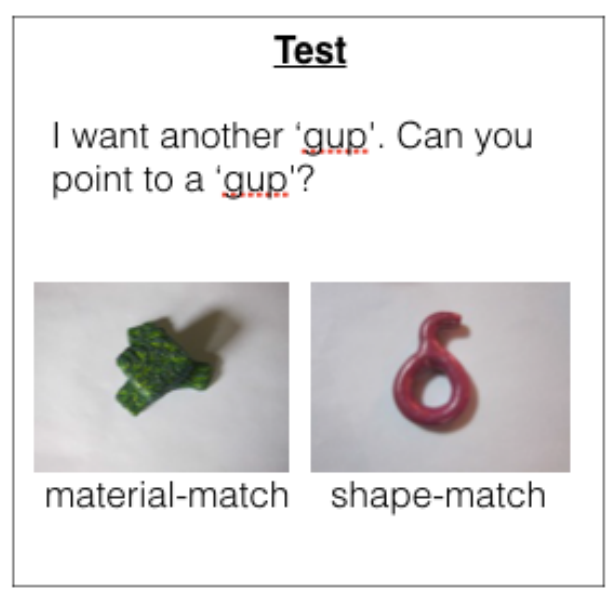

Figure 7. An example of a critical trial from Experiment 4. 
Materials and procedure. The materials and procedure were identical to the polysemy condition of Experiment 1 except that the standard object was not composed of the material that was presented in training (Figure 7). Thus, participants learned that the same novel word applied to a material (e.g., 'some gup') and an object (e.g., 'a gup'), but the object was not composed of the presented material. This was accomplished by shuffling the assignment of materials from Experiment 1 to different trials, e.g., such that the material from the first critical trial of Experiment 1 was presented on the second trial of Experiment 4 (but with the same standard object and test objects), the material from the second critical trial of Experiment 1 was presented on the third trial of Experiment 4, etc. As in Experiment 1, in the test phase of each critical trial, the participant was asked to extend the label for the standard object to either a material-match object (which matched the standard in material but not shape) or a shape-match object (which matched the standard in shape but not material, and did not match the initial material either): 'Okay, so now we know what [gups] are! And now it's your turn! I want another [gup]. Can you point to a [gup]?' Neither the material-match or shape-match objects was composed of the same material as the material presented in training.

Analyses. Our primary analyses, again using Bayesian logistic regression, examined whether participants from each age group chose the material-match object more often than chance (0.5). We also compared whether choices of the material-match object in Experiment 4 differed significantly from choices in the polysemy and unambiguous conditions of Experiment 1, which Experiment 4 was modeled directly after. Preliminary analyses did not find significant effects of age for children, or item order, and so these factors were dropped from subsequent models.

\section{Results}


Figure 2 depicts the proportion of critical trials in which participants selected the material-match object. As can be seen, children exhibited a shape bias, selecting the materialmatch object reliably less often than chance $(M=0.36[0.22,0.5]$, Beta $=-1.1(0.5), 95 \%$ Cr.I. [$2.3,-0.1])$. A follow-up analysis - combining the data from Experiment 4 with those from the polysemy and unambiguous conditions of Experiment 1 - determined that children in Experiment 4 were significantly less likely to select the material-match object than children in the polysemy condition of Experiment $1(M=0.70[0.56,0.82]$; Beta $=2.9(0.9), 95 \%$ Cr.I. $=[1.3,4.7])$. In contrast, children's selection of the material-match object in Experiment 4 did not significantly differ from that of children in the unambiguous condition of Experiment $1(M=0.27[0.14$, $0.40] ;$ Beta $=-1.0(0.9), 95 \%$ Cr.I. $=[-2.7,0.7])$. Together, these findings suggest that knowledge of a prior, material meaning for a new word affects children's extension of a new, object use of that word when it is possible for children to perceive the relation between the two word meanings: i.e., when the object is composed of the same material denoted by the word (Experiment 1), but not when it is composed of a different material (Experiment 4).

We found a broadly similar pattern of results in adults. Like children, adults in Experiment 4 also exhibited a shape bias, selecting the material-match object reliably less often than chance $(M=0.20[0.09,0.34]$, Beta $=-1.9(0.6), 95 \%$ Cr.I. $=[-3.2,-0.9])$. Also similar to children, a follow-up analysis combining adult data from Experiments 1 and 4 determined that adults in Experiment 4 were significantly less likely to select the material-match objects than adults in the polysemy condition of Experiment $1(M=0.89[0.75,1]$, Beta $=4.8(1.0), 95 \%$ Cr.I. $=[3.1,6.9])$, suggesting again that knowledge of a prior material meaning for a word more strongly affects extension of an object use of that word when the object is composed of the same material denoted by the word (which was the case in Experiment 1 but not Experiment 4). 
Interestingly, we also found that adults in Experiment 4 were significantly more likely to choose the material-match object than adults in the unambiguous condition of Experiment $1(M=0.02$ $[0,0.05]$, Beta $=-3.1(1.1), 95 \%$ Cr.I. $=[-5.5,-1.0])$, suggesting that for adults, hearing a word that has labeled a material used to refer to an object can still have some effect on subsequent extension of the object label, even when the object is composed of a different material than the word had previously labeled.

\section{General Discussion}

Because most words in natural language are polysemous, children will often be learning a new sense for a word that is already in their vocabulary, as opposed to an entirely new word. The present studies explored the implications of this basic fact for how children work out the structure of new word meanings. Consistent with previous findings, we found that when preschoolers and adults learn a new count noun for a solid object, they expect the word to be extended to other objects of the same shape (Landau et al., 1988; Smith et al., 1996). Critically, however, we also found that both preschoolers and adults will override a shape bias when they know that a word for a solid object can also be used to refer to the material that the object is composed of, as a mass noun. Specifically, using both a forced-choice (Experiments 1 and 2) and open-ended task (Experiment 3), we found that both children and adults typically extended a new word for an object by material when they had learned that the word could also refer to the material that made up the object (e.g., when the material was called 'some gup' and the object 'a gup'), even when they had encountered the material use after the object use (Experiment 2). In contrast, both adults and children in these experiments typically extended the object word by shape when it had not also been used to name the material (e.g., when the material was called 'some zev' and the object 'a gup'). Finally, we also found evidence for a boundary condition on 
when knowledge of a prior word use might affect beliefs about the extension of a new word meaning, by showing that if a word that has labeled a material ('some gup') has been used to label an object that is composed of a distinct material ('a gup') - such that the two uses of the word are unlikely to be perceived as related - neither children nor adults extend the object label by material, and instead adhere to a shape bias (Experiment 4$)$.

Together, our findings support the idea that children can use their knowledge of one sense of a polysemous word to constrain their hypotheses about how another sense of that word should be extended, allowing them to shift away from default heuristics like the shape bias in some cases. Further, our studies suggest that these effects of polysemy can arise regardless of the order in which learners encounter the senses of a polysemous word. Specifically, while Experiments 1 and 3 showed that a previously-encountered, material use of a polysemous word shifted children's and adults' extension of a subsequently-encountered, object use of that word toward material, Experiment 2 showed that exposure to a new, material use of a word led learners to revise their beliefs about the structure of a previously-encountered, object use of that word (i.e., such that they expected the object word to be extended by material). These findings suggest that when children encounter a new sense for a known word - which will often occur, given how ubiquitous polysemy is (Pustejovsky, 1995) - their interpretation of the new word sense will be affected by older senses, and their interpretation of the older senses may be affected by the new sense. Our findings suggest that polysemy may invite children to construe categories in new ways.

One intriguing possibility raised by our data is that polysemy may help children acquire adult-like extensional patterns for new word meanings. For example, the fact that children privileged material in their extension of a new object word ('a gup') when the same word had 
also labeled the object's material ('some gup') is consistent with how mature English speakers use words like 'glass' and 'tin': A brass cup cannot be called a 'glass', nor can a wooden box be called a 'tin'. Our findings thus suggest that the presence of material-object polysemy in a language may aid in the acquisition of the object senses of words like 'glass' and 'tin' by helping children quickly realize that material will play an important role in the structure of these word meanings; Critically, this insight would not be available to children if different word forms expressed the material and object senses of polysemous words like 'glass' and 'tin' in English. We expect that this benefit of polysemy documented here with material-object polysemy will apply in analogous ways to other forms of polysemy: e.g., a child that has learned that 'hammering' labels a functional use of a tool may expect that when 'hammer' is used to label an object, it will be extended by function rather than shape. Taken together with previous evidence that children can use predictable patterns of polysemy to spontaneously infer new senses of words (Srinivasan et al., 2017), our findings suggest that polysemy may facilitate word learning, providing a functional explanation for why polysemy is so ubiquitous in language (Rabagliati \& Srinivasan, in press; Srinivasan \& Rabagliati, 2015).

Although our findings suggest that polysemy can lead learners to expect a new object label to be extended by material, we would argue that this expectation - much like the shape bias - is best viewed as a first guess about the extension of a new word meaning, that will have to be adjusted as children accumulate additional evidence. This is important because, while the artifact senses of words like 'glass' and 'tin' are defined in part by their material, they label specific kinds of objects and are thus also defined by additional factors like function and shape; not all artifacts made of glass can be called 'glass', and for something to be called a drinking 'glass', it 
must support a specific function, which itself places constraints on its shape (e.g., it must be shaped so that it can hold liquid).

With this in mind, it is worth noting that both children and adults in the polysemy condition of Experiment 3 - who learned that the same word labeled a material and an object composed of that material - appeared to disregard shape altogether when extending the object labels, and instead extended these labels according to material alone. In particular, although the task of Experiment 3 was open-ended enough for participants in this condition to extend the object label to other objects that matched in both material and shape, no children and only one out of sixteen adults followed this strategy. Further, Experiment 1 found that, at least for children, this reliance on material did not reflect that they had only learned a single material meaning of the novel word, as opposed to separate material and object senses. One reason that participants may have ignored shape in their extension of the novel words is because they were not given specific information about the functions of the objects presented in training, limiting their hypotheses about the likely functional affordances - and shapes - of kind members (Kemler Nelson et al., 2000). An important task for future research will be to explore how children might integrate cues from polysemy with other kinds of information, like function, toward the end of constructing conventional word meanings.

Although we found broadly similar results in children and adults across our four studies, only children showed specific evidence in our task of having learned distinct material and object senses of the polysemous word in training, as opposed to treating the material and object presented in training as exemplars of a single material meaning. In particular, while both children and adults in Experiment 1 were more likely to extend a word for an object to a material-match object over a shape-match object when the word had previously labeled the material itself (in the 
polysemy condition, e.g., when the object was called 'a gup' and the material 'some gup'; Figure 2), only children were also more likely to extend the object label to the material-match object, as opposed to a portion of the material (material vs. object condition; Figure 2). Critically, if children had only learned a material meaning of the critical word - e.g., such that 'gup' labels a kind of material - they should have selected the portion of material at least as often as the material-match object, since both of these items consisted of the relevant material. The fact that children preferred the material-match object in this condition suggests that they had learned a distinct object sense of the polysemous word in training, and extended this word sense to the material-match object because it was a more prototypical object than the portion of material.

Given the performance of children described above, it was surprising that adults were at chance in selecting between the material-match object and the portion of material. We cannot rule out the possibility that, unlike children, adults only learned a single, material meaning of the word in training. By this account, after observing a word for a material (e.g., 'some gup'), adults may have construed the object ('a gup') as another exemplar of the material, as opposed to as an object made from the material. However, as noted before, previous studies indicate that both children and adults are unlikely to interpret a word as referring to an unindividuated substance when it is embedded in count syntax and applied to a solid, complex, regularly-shaped object (Barner \& Snedeker, 2006; Imai \& Gentner, 1997; Li, et al., 2009; Prasada et al., 2002; Soja, 1992; Soja, Carey, \& Spelke, 1991; Subrahmanyam et al., 1999), as was the case in our studies. An alternative possibility is that adults, like children, learned two distinct senses of the novel polysemous words: one sense that referred to the material, as a mass noun ('some gup'), and the other that referred to an individual made from the material, as a count noun ('a gup'). By this account, the difference between adults and children at test may have been that adults were more 
likely than children to construe the portion of material as an individual, making them more likely to consider it to be 'a gup' - similar to how a portion of water can be called 'a water' or a portion of coffee can be called 'a coffee'. Future studies should explore children's and adults' ability to generate portioning interpretations of count nouns, a topic that has yet to receive much study (Copestake \& Briscoe, 1995; Frisson \& Frazier, 2005).

Importantly, our studies provide insight into the conditions in which children's knowledge of one meaning of a word might affect their beliefs about another meaning of that word. In particular, when children were unlikely to perceive the object meaning of a word as being related to the material meaning of that word - because the word for the material was used to label an object made from a different material (Experiment 4) - they appeared to treat the object label similarly to any new count noun for a solid object, and extend it by shape rather than by material. This finding suggests that the effects of polysemy documented in the present studies may be contingent on children's beliefs that two uses of a word are related. By this account, while a known sense of a polysemous word should affect children's interpretation of a new sense of that word (e.g., 'glass' material and 'glass' artifact) because children can perceive the two senses to be related, a known homophone should not affect children's interpretation of a new homophone (e.g., baseball 'bat' and animal 'bat'), because children can perceive the two meanings to be unrelated. Our findings could also be explained by the attentional learning account of Yoshida and Smith (2003) if, under that account, hearing a material name used for an object only biases children to attend to the material dimension of that object - and extend the object label by material - when the object is made from the specific material associated with the material name. 
Finally, while prior research has focused on how children use shared labels as invitations to group items into common categories (Gelman \& Brandone, 2010; Waxman \& Markow, 1995), our findings show that children can recognize situations in which a shared label is being used to refer to items from different categories and use this as a basis for constructing relations between those categories. For instance, rather than leading children to expect 'glass' material and drinking 'glasses' to be members of a common category, polysemy may invite children to understand how these distinct categories relate to and constrain one another. More generally, by attending to the widespread and systematic polysemy in their language, children may be able to draw a variety of inferences about the world. For example, children who have learned that the same words can label tools and the functions those tools support (e.g., 'hammer', 'shovel', 'rake') could reason that when a word for a function is re-used to label an object, the object is likely to belong to a category that is defined in part by that function. Thus, children could reason that an object that is called a 'dax' that supports a function called 'daxing' has probably been designed for that function, and that all 'daxes' should support this function, just as 'hammers' are designed for 'hammering' and all 'hammers' should support 'hammering'. Viewed in this way, polysemy may support cognitive development more broadly. 


\section{Context of the Research}

Most common words are polysemous, and possess multiple related senses. The present study is part of a broader research program that aims to understand why this type of ambiguity is so prevalent in language, and what it reveals about lexical and conceptual development. Our key finding — that learning one meaning for a word influences how children learn its other senses suggests that polysemy may facilitate lexical development, and thus potentially provides a functional explanation for the prevalence of polysemy: It is easier for children to learn one word with multiple related senses, than to learn the meanings of multiple unambiguous words. This motivates a re-thinking of dominant theories of word learning, since according to many of these theories, children assume that words will label a single meaning and should thus have difficulty learning polysemous words. Further research is needed to characterize the different ways in which polysemy may facilitate word learning and shape conceptual development, beyond the findings reported here. For example, polysemy may affect not only how children extend new word meanings, but also how they encode and retain these word meanings, and how they process them on-line. These ideas also raise questions about how children's understanding of polysemy relates to their world knowledge, and their ability to use the linguistic context to interpret novel uses of words. Finally, it will be important to understand individual differences in how children learn and use new word senses, including differences among typically-developing children and children with developmental disorders. 


\section{References}

Apresjan, J.D. (1974). Regular polysemy. Linguistics, 1 (2), 5-32.

Barner, D., \& Snedeker, J. (2005). Quantity judgments and individuation: Evidence that mass nouns count. Cognition, 97(1), 41-66. http://doi.org/10.1016/j.cognition.2004.06.009

Barner, D., \& Snedeker, J. (2006). Children's early understanding of mass-count syntax: Individuation, lexical content, and the number asymmetry hypothesis. Language Learning and Development, 2(3), 163-194.

Beveridge, M., \& Marsh, L. (1991). The influence of linguistic context on young children's understanding of homophonic words. Journal of Child Language, 18(2), 459-467. http://doi.org/10.1017/S0305000900011168

Bloom, P. (2002). How children learn the meanings of words. Cambridge, MA: MIT Press.

Booth, A. E., \& Waxman, S. R. (2002). Word learning is "smart": evidence that conceptual information affects preschoolers' extension of novel words. Cognition, 84(1), B11-B22. http://doi.org/10.1016/S0010-0277(02)00015-X

Booth, A. E., Waxman, S. R., \& Huang, Y. T. (2005). Conceptual information permeates word learning in infancy. Developmental Psychology, 41(3), 491-505. http://doi.org/10.1037/0012-1649.41.3.491

Bowerman, M. (1983). Hidden meanings: The role of covert conceptual structures in children's development of language. In D. R. Rogers \& J. A. Sloboda (Eds.), The acquisition of symbolic skills (pp. 445-470). New York: Plenum Press.

Campbell, R. N., \& MacDonald, T. B. (1983). Text and context in early language comprehension. In Early childhood development and education (pp. 115-126).

Casenhiser, D. M. (2005). Children's resistance to homonymy: an experimental study of pseudohomonyms. Journal of Child Language, 32(2), 319-343. 
http://doi.org/10.1017/S0305000904006749

Clark, E. V. (1982). The young word maker: a case study of innovation in the child's lexicon. Language Acquisition: The State of the Art, (1), 390-425.

Copestake, A., \& Briscoe, T. (1995). Semi-productive polysemy and sense extension. Journal of Semantics, 12(1), 15-67.

Dautriche, I., Chemla, E., \& Christophe, A. (2016). Word Learning: Homophony and the Distribution of Learning Exemplars. Language Learning and Development, 12(3), 231-251. http://doi.org/10.1080/15475441.2015.1127163

Diesendruck, G., Markson, L., \& Bloom, P. (2003). Children 'S Reliance on Creator ' S Intent in Extending Names for Artifacts. Psychological Science, 14(2), 164-168.

Doherty, M. J. (2004). Childrens difficulty in learning homonyms. Journal of Child Language, 31(1), 203-214. http://doi.org/10.1017/S030500090300583X

Frazier, L., \& Rayner, K. (1990). Taking on semantic commitments: Processing multiple meanings vs. multiple senses. Journal of Memory and Language, 29(2), 181-200. http://doi.org/10.1016/0749-596X(90)90071-7

Frisson, S., \& Frazier, L. (2005). Carving up word meaning: Portioning and grinding. Journal of Memory and Language, 53(2), 277-291. http://doi.org/10.1016/J.JML.2005.03.004

Gelman, S. a., \& Bloom, P. (2000). Young children are sensitive to how an object was created when deciding what to name it. Cognition, 76(2), 91-103. http://doi.org/10.1016/S00100277(00)00071-8

Gelman, S. A., \& Brandone, A. C. (2010). Fast-Mapping Placeholders: Using Words to Talk About Kinds. Language Learning and Development, 6(3), 223-240.

http://doi.org/10.1080/15475441.2010.484413 
Goodman, N. (1983). Fact, fiction, and forecast. Harvard University Press.

Harper, D. (2001). Online etymology dictionary. Retrieved from http://www.etymonline.com

Imai, M., \& Gentner, D. (1997). A cross-linguistic study of early word meaning: universal ontology and linguistic influence. Cognition, 62(2), 169-200. http://doi.org/10.1016/S00100277(96)00784-6

Jones, S. S., Smith, L. B., \& Landau, B. (1991). Object Properties and Knowledge in Early Lexical Learning. Child Development, 62(3), 499-516. http://doi.org/10.1111/j.14678624.1991.tb01547.x

Kemler Nelson, D. G., Russell, R., Duke, N., \& Jones, K. (2000). Two-year-olds will name artifacts by their functions. Child Development, 71(5), 1271-1288. http://doi.org/doi:10.1111/1467-8624.00228

Landau, B., Smith, L., \& Jones, S. (1988). The importance of shape in early lexical learning. Cognitive Development, 3(3), 299-321.

Li, P., Dunham, Y., \& Carey, S. (2009). Of substance: The nature of language effects on entity construal. Cognitive Psychology, 58(4), 487-524. http://doi.org/10.1016/j.cogpsych.2008.12.001

Markman, E. (1990). Constraints Children Place on Word Meanings. Cognitive Science, 14(1), 55-77. http://doi.org/10.1016/0364-0213(90)90026-S

Markman, E. (1991). Categorization and naming in children: Problems of induction. Cambridge: MIT Press.

Mazzocco, M. M. (1997). Children's interpretations of homonyms: a developmental study. Journal of Child Language, 24(2), 441-467.

Parks, R., Ray, J., \& Bland, S. (1998). Wordsmyth English dictionary-thesaurus. Retrieved from 
http://www.wordsmyth.net/

Peters, A., \& Zaidel, E. (1980). The acquisition of homonymy. Cognition, 8(2), 187-207. http://doi.org/10.1016/0010-0277(80)90012-8

Prasada, S., Ferenz, K., \& Haskell, T. (2002). Conceiving of entities as objects and as stuff. Cognition, 83(2), 141-165. http://doi.org/10.1016/S0010-0277(01)00173-1

Pustejovsky, J. (1995). The generative lexicon. Cambridge: MIT Press.

Quine, W. (1969). 0.(1960) Word and object. Cambridge, Mass.

Rabagliati, H., Marcus, G. F., \& Pylkkänen, L. (2013). Top-Down Influence in Young Children ' s Linguistic Ambiguity Resolution. Developmental Psychology, 49(6), 1076-1089. http://doi.org/10.1037/a0026918

Rabagliati, H., Marcus, G., \& Pylkkänen, L. (2010). Shifting senses in lexical semantic development. Cognition, 117(1), 17-37.

Rabagliati, H., \& Srinivasan, M. (n.d.). Word Senses. In C. Cummins (Ed.), Oxford Handbook of Experimental Semantics and Pragmatics. Oxford University Press.

Rodd, J. M., Gaskell, M. G., \& Marslen-Wilson, W. D. (2004). Modelling the effects of semantic ambiguity in word recognition. Cognitive Science, 28(1), 89-104. http://doi.org/10.1016/J.COGSCI.2003.08.002

Smith, L. B. (2000). How to learn words: An associative crane. In Breaking the word learning barrier. Oxford University Press.

Smith, L. B., Jones, S. S., Landau, B., Gershkoff-Stowe, L., \& Samuelson, L. (2002). Object name Learning Provides On-the-Job Training for Attention. Psychological Science, 13(1), 13-19. http://doi.org/10.1111/1467-9280.00403

Smith, L. B., \& Samuelson, L. (2006). An attentional learning account of the shape bias: Reply 
to Cimpian and Markman (2005) and Booth, Waxman, and Huang (2005). Developmental Psychology, 42(6), 1339-1343. http://doi.org/10.1037/0012-1649.42.6.1339

Smith, L., Jones, S., \& Landau, B. (1996). Naming in young children: A dumb attentional mechanism? Cognition, 60(2), 143-171.

Smith, M. (1933). Grammatical errors in the speech of pre-school children. Child Development, 4(2), 183-190.

Soja, N. N. (1992). Inferences about the meanings of nouns: The relationship between perception and syntax. Cognitive Development, 7(1), 29-45. http://doi.org/10.1016/08852014(92)90003-A

Soja, N. N., Carey, S., \& Spelke, E. S. (1991). Ontological categories guide young children's inductions of word meaning: Object terms and substance terms. Cognition, 38(2), 179-211. http://doi.org/10.1016/0010-0277(91)90051-5

Srinivasan, M., Al-Mughairy, S., Foushee, R., \& Barner, D. (2017). Learning language from within: Children use semantic generalizations to infer word meanings. Cognition, 159. http://doi.org/10.1016/j.cognition.2016.10.019

Srinivasan, M., \& Rabagliati, H. (2015). How concepts and conventions structure the lexicon: Cross-linguistic evidence from polysemy. Lingua, 157, 124-152.

Srinivasan, M., \& Snedeker, J. (2011). Judging a book by its cover and its contents: The representation of polysemous and homophonous meanings in four-year-old children. Cognitive Psychology, 62(4), 245-272.

Srinivasan, M., \& Snedeker, J. (2014). Polysemy and the Taxonomic Constraint: Children's Representation of Words that Label Multiple Kinds. Language Learning and Development, 10(2). http://doi.org/10.1080/15475441.2013.820121 
Subrahmanyam, K., Landau, B., \& Gelman, R. (1999). Shape, Material, and Syntax: Interacting Forces in Children's Learning in Novel Words for Objects and Substances. Language and Cognitive Processes, 14(3), 249-281. http://doi.org/10.1080/016909699386301

Waxman, S. R., \& Markow, D. B. (1995). Words as Invitations to Form Categories: Evidence from 12- to 13-Month-Old Infants. Cognitive Psychology, 29(3), 257-302. http://doi.org/10.1006/COGP.1995.1016

Yoshida, H., \& Smith, L. B. (2003). Known and Novel Noun Extensions : Attention at Two Levels of Abstraction, 74(2), 564-577. 